\title{
New findings on the prototypical Of?p stars ${ }^{\star}$
}

\author{
Y. Nazé ${ }^{1, \star \star}$, A. ud-Doula ${ }^{2}$, M. Spano ${ }^{3}$, G. Rauw ${ }^{1, \star \star}$, M. De Becker ${ }^{1,4}$, and N. R. Walborn ${ }^{5}$ \\ ${ }^{1}$ GAPHE, Département AGO, Université de Liège, Allée du 6 Août 17, Bât. B5C, B4000 Liège, Belgium \\ e-mail: naze@astro.ulg.ac.be \\ 2 Penn State Worthington Scranton, 120 Ridge View Drive, Dunmore, PA 18512, USA \\ 3 Observatoire de Genève, Université de Genève, 51 Chemin des Maillettes, 1290 Sauverny, Switzerland \\ 4 Observatoire de Haute-Provence, 04870 Saint-Michel l'Observatoire, France \\ 5 Space Telescope Science Institute ${ }^{\star \star \star}, 3700$ San Martin Drive, Baltimore, MD 21218, USA
}

Received 1 March 2010 / Accepted 7 June 2010

\section{ABSTRACT}

\begin{abstract}
Aims. In recent years several in-depth investigations of the three prototypical Of?p stars were undertaken. These multiwavelength studies revealed the peculiar properties of these objects (in the X-rays as well as in the optical): magnetic fields, periodic line profile variations, recurrent photometric changes. However, many questions remain unsolved.

Methods. To clarify some of the properties of the Of?p stars, we have continued their monitoring. A new XMM-Newton observation and two new optical datasets were obtained.

Results. Additional information about the prototypical Of?p trio has been found. HD 108 has now reached its quiescent, minimumemission state for the first time in 50-60 yr. The échelle spectra of HD 148937 confirm the presence of the 7d variations in the Balmer lines and reveal similar periodic variations (though of lower amplitudes) in the He I $\lambda 5876$ and He II $\lambda 4686$ lines, underlining its similarities with the other two prototypical Of?p stars. The new XMM-Newton observation of HD 191612 was taken at the same phase in the line modulation cycle, but at a different orbital phase from previous data. It clearly shows that the X-ray emission of HD 191612 is modulated by the 538d period and not by the orbital period of $1542 \mathrm{~d}$ - it is thus not of colliding-wind origin. The phenomenon responsible for the optical changes appears also at work in the high-energy domain. There are problems however: our MHD simulations of the wind magnetic confinement predict both a harder X-ray flux of a much larger strength than what is observed (the modelled differential emission measure peaks at 30-40 MK, whereas the observed one peaks at $2 \mathrm{MK}$ ) and narrow lines (hot gas moving with velocities of $100-200 \mathrm{~km} \mathrm{~s}^{-1}$, whereas the observed full width at half maximum is $\sim 2000 \mathrm{~km} \mathrm{~s}^{-1}$ ).
\end{abstract}

Key words. X-rays: stars - stars: individual: HD 108 - stars: individual: HD 148937 - stars: individual: HD 191612 stars: emission-line, Be

\section{Introduction}

The presence of C III $\lambda 4650 \AA$ in emission with a strength comparable to the neighbouring $\mathrm{N}$ III lines is not a usual feature of $\mathrm{O}$-star spectra. In the past, stars displaying this feature were classified by Walborn (1972) in a separate category, dubbed Of?p. In recent years, this category has attracted a lot of attention, as one can see from the numerous publications (Walborn et al. 2003, 2004; Donati et al. 2006; Howarth et al. 2007; Hubrig et al. 2008; Nazé et al. 2001, 2004, 2007, 2008a). They dealt with the peculiar properties of these stars (for a summary see Nazé et al. 2008b) and led to a modification of the definition of these stars. The Of?p phenomenon now covers stars with recurrent spectral variations (in the Balmer, He I, C III, Si III lines), strong C III $\lambda 4650 \AA$ in emission (at least at some phases), narrow P Cygni or emission components in the Balmer hydrogen lines and HeI lines (at least at some phases), and UV wind

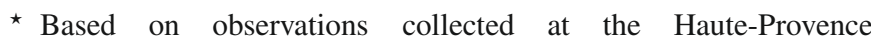
Observatory, at the La Silla and San Pedro Mártir Observatories, and with XMM-Newton, an ESA Science Mission with instruments and contributions directly funded by ESA Member States and the USA (NASA).

$\star \star$ Research Associate FRS-FNRS.

$\star \star \star$ Operated by the Association of Universities for Research in Astronomy, Inc., under NASA contract NAS5-26555. lines weaker than for typical Of supergiants (Nazé et al. 2008a; Walborn et al. 2010). Stars that appear otherwise normal but display strong C III $\lambda 4650$ emission are now called "Ofc" stars (Walborn et al. 2010).

The three prototypical Of?p stars are HD 108, HD 148937 , and HD 191612. They are the most studied objects of that category, though some open questions still remain.

HD 108 displays correlated photometric, Balmer, and He I line profile variations: the star is brighter when the emission lines are stronger (Nazé et al. 2001; Nazé et al. 2008b). The variations appear in a recurrent mode, with a probable time scale of about 55 years (Nazé et al. 2006). The minimum emission state was not yet reached in 2004 (Nazé et al. 2004), which prevented a precise evaluation of the recurrence time. Recently, an additional observational result enriched the picture of HD 108: the detection of a strong magnetic field (observed strength is $100-150 \mathrm{G}$, corresponding to a potential dipolar field of 0.5-2 kG Martins et al. 2010).

The behaviour of HD 191612 appears very similar to that of HD 108, but with a shorter, well-defined period of 537.6d (Howarth et al. 2007, and references therein). A strong magnetic field $(-220 \pm 38 \mathrm{G})$ was detected for this star by Donati et al. (2006), who further proposed an oblique magnetic rotator model for the star. Following these authors, the long period would result from magnetic braking of the stellar rotation (for an analysis 
of these effects see ud-Doula et al. 2009). Studies are now under way to check the exact geometry of the magnetic field (Wade, private communication). Finally, it should be noted that this star has a lower-mass companion in a $1542 \mathrm{~d}$ orbit (Howarth et al. 2007).

HD 148937 does not display the large photometric changes and line profile variations like the two other objects of this study. Instead, changes of limited amplitude (the peak's amplitude varyies by a few percents vs several $100 \%$ for the other prototypical Of?p stars) were observed for the $\mathrm{H} \alpha$ line, with a possible period of about $7 \mathrm{~d}$, during a long-term monitoring campaign (Nazé et al. 2008a), but the temporal sampling of the optical dataset was not optimized for finding such a short period and the resultant value may therefore be subject to large errors. In addition, no change was detected in the low-resolution data for other spectral lines, which differs from what is observed in HD 108 and HD 191612. Finally, a tentative detection of a magnetic field was reported for this star by Hubrig et al. $\left(2008, B_{z}=-276 \pm 88 \mathrm{G}\right)$, but a spectro-polarimetric monitoring throughout the $7 \mathrm{~d}$ cycle is clearly needed to ascertain the strength and geometry of the field (it is currently under way, Wade, private communication).

In addition to dedicated optical campaigns, several XMM-Newton observations of these three objects were obtained. The derived properties are remarkably similar: overluminosities with respect to the "canonical" $L_{X} / L_{\mathrm{BOL}}=10^{-7}$ relation, soft spectra, broad X-ray lines (Nazé et al. 2004, 2007, 2008a). Monitoring HD 191612 further revealed small variations of the $\mathrm{X}$-ray flux. However, as all data were taken during the same orbital cycle and the same 538d cycle, the exact cause for these changes remained unclear.

Though the published studies have led to the characterization of many aspects of these Of?p stars, several questions still remain unanswered - usually, these questions are different for the different objects (e.g. on the variations of the X-rays of HD 191612, on the evolution of the optical spectra of HD 108, or on the variation timescale of HD 148937), due to the inhomogeneous datasets reported up to now. In the past few years we continued our monitoring of these three peculiar objects, with the objective of characterizing and better understanding their properties. This paper reports the results of this campaign. In Sect. 2 we present the datasets and in Sect. 3 the results, in Sect. 4 we summarize the new information.

\section{Observations and data reduction}

\subsection{Aurélie data}

Since 2004, when optical spectra of HD 108 were last published (Nazé et al. 2004), we have continued the monitoring of the star with the Aurélie spectrograph mounted on the $1.52 \mathrm{~m}$ telescope of the Haute-Provence Observatory (OHP, France). The detector was a thin back-illuminated CCD composed of $2048 \times 1024$ pixels of size $13.5 \mu \mathrm{m}^{2}$. We used the grating \#3, reaching a resolving power between 8000 and 10000 in the blue and red part of the spectrum, respectively. With this instrumental setup we obtained spectra in the 4450-4900, , 5480-5920 , and 6340-6780 ranges. Exposure times were typically $20-40 \mathrm{~min}$, resulting in a signal-to-noise ratio of about 200 on average. The data were reduced in a standard way using MIDAS. The wavelength calibration was done using ThAr exposures taken immediately before/after the stellar spectrum, and the spectra were normalised using low-degree polynomials.

An additional échelle spectrum obtained in June 2009 at San Pedro Mártir with the Espresso spectrograph was also made available to us by P. Eenens and L. Mahy. This dataset has been added to the analysis and discussion.

\subsection{Coralie échelle spectra}

Twenty échelle spectra of HD 148937 were obtained in 2008 May 8-21 with the $1.2 \mathrm{~m}$ Euler Swiss telescope at La Silla (Chile), equipped with the Coralie spectrograph and an EEV $2 \mathrm{k} \times 2 \mathrm{k}$ CCD with pixel size $15 \mu \mathrm{m}^{2}$. The Coralie instrument is an improved version of the Élodie spectrograph (Baranne et al. 1996). These observations covered the spectral range 3850-6890 A with a resolving power of 55000 . During the first week, two spectra per night were taken; during the second week, one spectrum per night was taken. The integration times were fixed to $30 \mathrm{~min}$ (except for the first two exposures, which were each $20 \mathrm{~min}$ long) and the typical $\mathrm{S} / \mathrm{N}$ ratio at $5000 \AA$ was 150 . The data were first reduced with the Coralie pipeline and then normalised using low-degree polynomials. A telluric correction was applied for $\mathrm{H} \alpha$ and $\mathrm{He}$ I $\lambda 5876$ using the telluric spectrum of Hinkle et al. (2000) and the task telluric within IRAF.

\subsection{XMM-Newton observation}

HD 191612 was observed anew by XMM-Newton on 2008 April 2 (Rev. 1523, ObsID 050068201, PI Nazé), for a total exposure time of $24 \mathrm{ks}$. The instrumental configuration, processing, and extraction regions were identical to the first four observations of the source (Nazé et al. 2007). We processed the data with the Science Analysis System (SAS) software, version 8.0; further analysis was performed using the FTOOLS tasks and the XSPEC software v 11.2.0. No high background episode (due to soft protons) was detected at high-energies during this exposure.

In addition to European Photon Imaging Camera (EPIC) data, XMM-Newton provides high-resolution spectra thanks to the Reflection Grating Spectrometer (RGS) instruments. The RGS data were reduced with the SAS and then combined with the previous observations following the procedure outlined in Nazé et al. (2007). However, the improvement in signal-to-noise of the combined spectrum is not important because all observations are of similar duration. We therefore have no new results to report on the high-resolution spectrum of HD 191612, a quantitative leap in quality awaiting the advent of more sensitive observatories.

\section{New results on Of?p stars}

\section{1. $H D 108$}

The spectrum of HD 108 has been followed intensively since 1986 (Nazé et al. 2001, 2004). While some (purely photospheric) lines such as He II $\lambda 4542$ remain constant, the strength of the Balmer, He I (e.g. at $\lambda \lambda 4471,4713,6678)$, Si III (e.g. at $\lambda 4552)$ and C III $\lambda \lambda 4650$ lines have decreased with time, with profiles changing from pure emissions or P Cygni profiles to strong absorptions or greatly reduced emissions, as illustrated by Fig. 1. These lines thus appear as a blend of a constant photospheric absorption and a varying emission component. A similar change was observed about 55 years ago (Nazé et al. 2001, 2006), but due to the lack of detailed monitoring in the past - often, only a global idea of the profile (emission vs absorption) was recorded - the timescale of the variations could not be precisely assessed and some even questioned the recurrence of the phenomenon. It is therefore of the utmost importance to see how the lines evolve and if quiescence is reached. 
Y. Nazé et al.: New findings on the prototypical Of?p stars
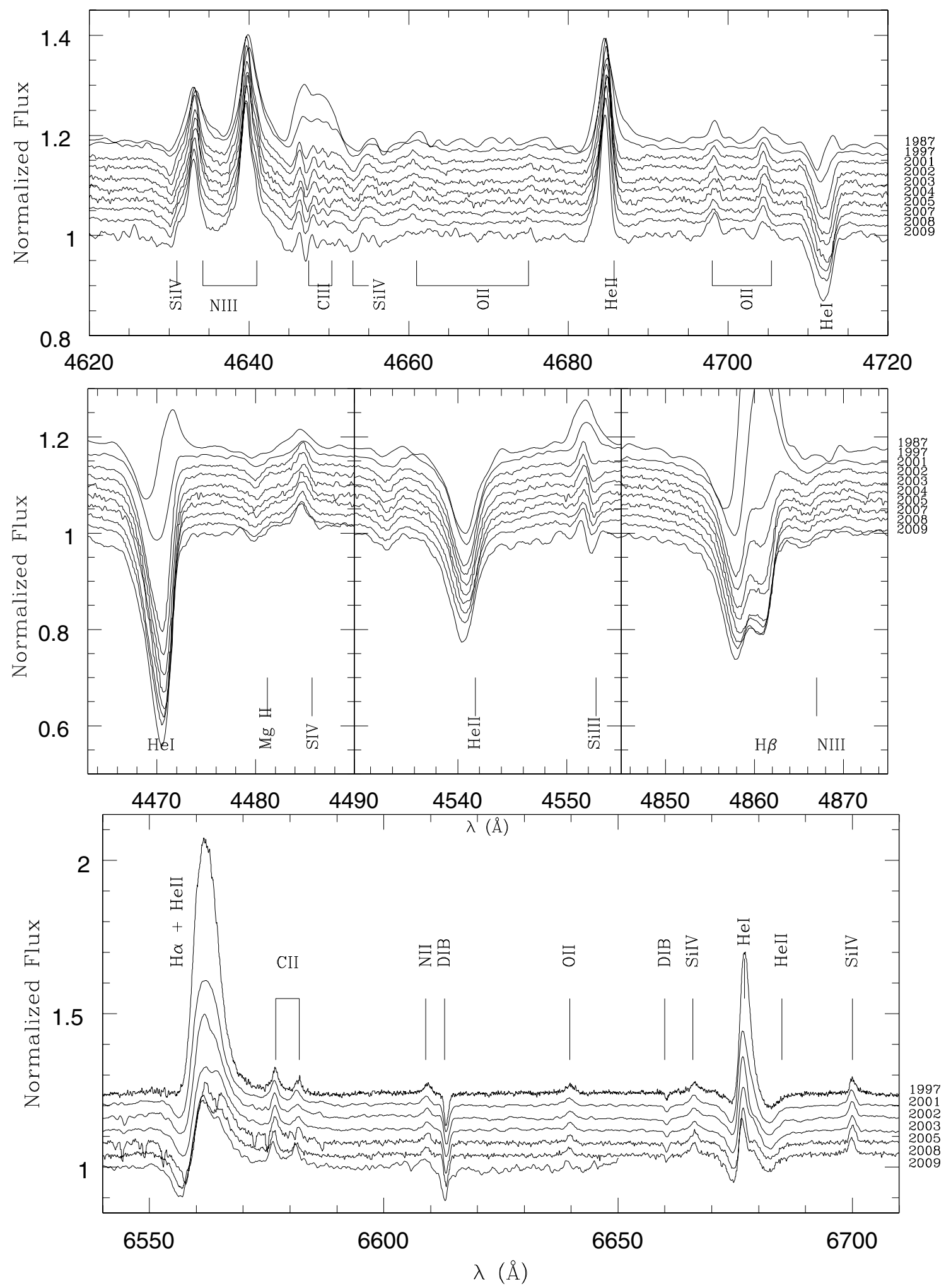

Fig. 1. Evolution of the line profiles in recent years for HD 108.

Though the emission component had nearly disappeared these last years (Nazé et al. 2004), the emissions continued to decline until 2004. Now, HD 108 has finally reached its quiescent state because the data taken between 2005 and 2009 are very similar (Figs. 1 and 2). Based on past behaviour (Nazé et al. 2001), it is expected that the emission will get stronger again in the future, as is seen in HD 191612. Note however that for HD 191612 the quiescent state occurs during one-third of the cycle, which would correspond to about 18 years for HD 108 . Assuming that the variations are due to an oblique magnetic rotator configuration, this quiescence timescale would depend on the exact geometry of the system though, which may be 


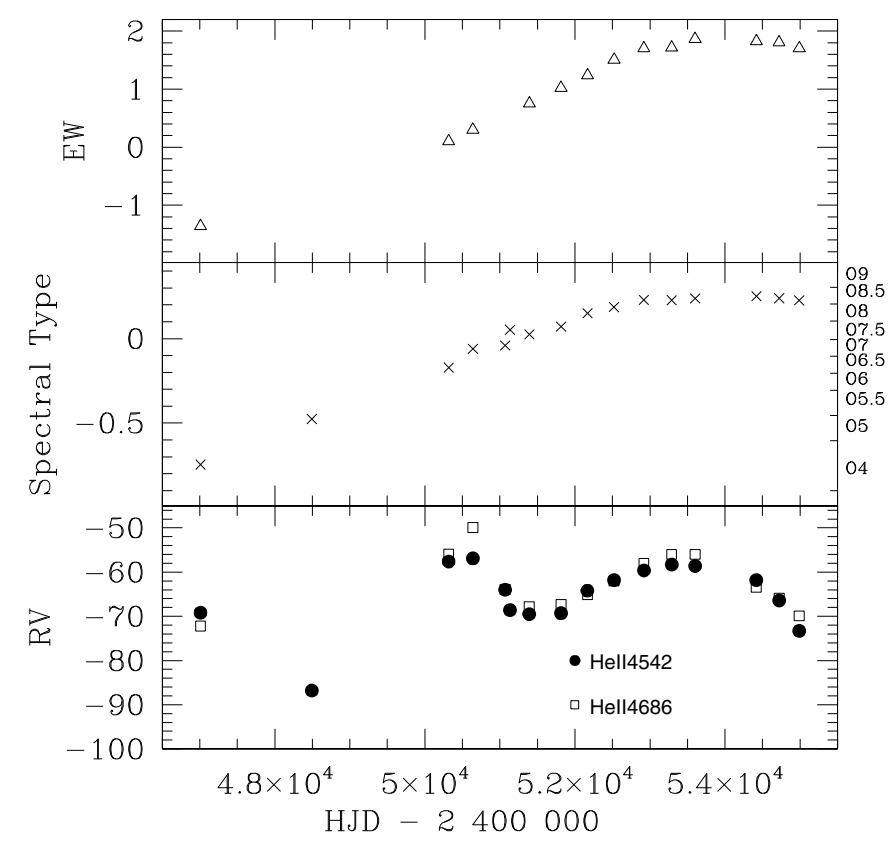

Fig. 2. Evolution as a function of time of a few quantities related to HD 108. Upper panel: $E W$ (in $\AA$, in the range 4845-4870 $\AA$ ) of $\mathrm{H} \beta$. Middle panel: spectral type criterion $\log \left(\frac{E W_{\mathrm{He}[4711}}{E W_{\mathrm{Hel} 4442}}\right)$. Lower panel: RVs (in $\mathrm{km} \mathrm{s}^{-1}$ ) of He II $\lambda \lambda 4542,4686$.

different for the two objects despite their obvious similarities. From the literature it is only known that $\mathrm{H} \beta$ remained in absorption during about 10 years, while He I $\lambda 4471$ did so during several decades (Nazé et al. 2001). However, the exact evolution was not recorded and it cannot be concluded from the older data how long the lines remained constant, i.e. in a quiescent state.

\section{2. $H D 148937$}

Previously, a long-term monitoring (three years, with monthly observations) provided an extensive set of low-resolution spectra. These data revealed the variability of three lines: $\mathrm{H} \alpha, \mathrm{H} \beta$, and He II $\lambda 4686$ (Nazé et al. 2008a). However, this variability was of low amplitude: the equivalent widths ( $E W \mathrm{~s})$ measured for $\mathrm{H} \alpha$, the most variable line, display a dispersion which, though it was 10 times larger than that observed for the neighbouring narrow diffuse interstellar band (DIB), correspond to a change of only $20 \%$ in $E W$ (it also corresponds to a variation in the peak's amplitude of only $7 \%$ between the two extreme profiles), whereas the other prototypical Of?p stars have $\mathrm{H} \alpha$ lines varying from absorption to emission, with $E W$ s and peak amplitudes varying by $\gg 100 \%$. Moreover, the He I and C III lines seemed relatively constant, a behaviour different from that of HD 108 and HD 191612 - though subtle changes may have remained hidden in such low-resolution data. A period search further revealed a period of $7.031 \pm 0.003 \mathrm{~d}$ when we studied the line profile variations of $\mathrm{H} \alpha$ closely. However, the data sampling, aimed at studying monthly-to-yearly variations, was not adequate for detecting such a short period, which thus required confirmation with a more intense temporal sampling. A new, short-term monitoring was therefore undertaken, yielding 20 high-resolution spectra over two weeks.

For consistency, the radial velocities and $E W \mathrm{~s}$ were estimated as in Nazé et al. (2008a); they are given in Table 1.
Table 1. Mean and dispersion of the measured RVs and EWs for HD 148937.

\begin{tabular}{lrr}
\hline \hline Line & $\begin{array}{r}\text { RV } \\
\left(\mathrm{km} \mathrm{s}^{-1}\right)\end{array}$ & $\begin{array}{r}E W \\
(\AA)\end{array}$ \\
\hline $\mathrm{H} \gamma$ & & $1.467 \pm 0.044$ \\
$\mathrm{He}$ II $\lambda 4686$ & $-14.4 \pm 2.7$ & $-0.697 \pm 0.064$ \\
$\mathrm{H} \beta$ & & $0.931 \pm 0.061$ \\
$\mathrm{C} \mathrm{IV} \lambda 5812$ & $-33.6 \pm 2.3$ & $0.231 \pm 0.013$ \\
$\mathrm{He}$ I $\lambda 5876$ & & $0.416 \pm 0.038$ \\
$\mathrm{H} \alpha$ & & $-0.871 \pm 0.251$ \\
$\mathrm{DIB} \lambda 6613$ & $-3.3 \pm 0.5$ & $0.143 \pm 0.010$ \\
\hline
\end{tabular}

While the CIV and DIBs display constant $E W \mathrm{~s}$, the Balmer, He II $\lambda$ 4686, and He I $\lambda 5876$ lines show a larger dispersion. When plotted against time (Fig. 3), obvious modulations are detected. $\mathrm{H} \alpha$ and $\mathrm{He}$ II $\lambda 4686$ lines display maximum emission when $\mathrm{H} \beta, \mathrm{H} \gamma$, and $\mathrm{He} \mathrm{I} \lambda 5876$ present a minimum absorption.

The variability detected when looking at $E W \mathrm{~s}$ is confirmed by the temporal variance spectrum (TVS, see Fullerton et al. 1996, and Fig. 4), which compares the variations between spectra with the scatter expected from random noise in each wavelength bin. Note that the variability has not the same amplitude for all lines of a given element: for the Balmer lines, the TVS is very significant for $\mathrm{H} \alpha$, significant for $\mathrm{H} \beta$, and barely significant for $\mathrm{H} \gamma$. This might explain why the variability of, e.g., He I $\lambda 4471$ is not detected - its amplitude, expected to be lower than for He I $\lambda 5876$, is certainly hidden by the noise. Note that no significant variation of the C III $\lambda 4650$ lines is detected.

Periodograms were calculated for the 20-exposures dataset using the techniques of Heck et al. (1985, see remarks in Gosset et al. 2001) in the wavelength interval where lines vary significantly (for details about the method see Rauw et al. 2001). The results are shown in Fig. 5: the highest peak is located at a frequency of $0.1315 \mathrm{~d}^{-1}, 0.1353 \mathrm{~d}^{-1}, 0.1373 \mathrm{~d}^{-1}$, and $0.1397 \mathrm{~d}^{-1}$ for He II $\lambda$ 4686, H $\beta$, He I $\lambda$ 5876, and $\mathrm{H} \alpha$, respectively. These frequencies translate into a period of $7.16-7.60 \pm 0.40 \mathrm{~d}$. The relatively large error compared to the old dataset simply stems from the different observational timespans (two weeks vs. 2.5 years), while the period differences from one line to the other have two origins: the noise, which more affects the fainter lines such as He I $\lambda$ 5876, and the different amplitudes of the variations (the signal is indeed more difficult to catch for low amplitude changes, e.g. for He I $\lambda$ 5876). The $\mathrm{H} \alpha$ line, which is both strong and the most variable line, yields the most reliable period, i.e. $7.16 \pm 0.40 \mathrm{~d}$ from the new dataset only. Taking the errors into account ${ }^{1}$, this agrees with the results reported by Nazé et al. (2008a). The 7d period was thus not an artefact from our inadequate time sampling. HD 148937 therefore appears quite similar to the two other Galactic Of?p stars, though with a shorter period, a smaller amplitude of the variations, and with the sole exception of the apparent constancy in the C III lines. A spectropolarimetric monitoring should be undertaken to see if this could be related to e.g. a low inclination of the star's rotation axis.

\footnotetext{
1 Note that combining the two datasets is not possible due to the very different spectral resolutions $(R=2300$ vs. 55000$)$. As the best period estimate comes from the longest time coverage, the value of $7.031 \pm 0.003 \mathrm{~d}$ from Nazé et al. (2008a) should be considered as the best measurement on an absolute scale.
} 

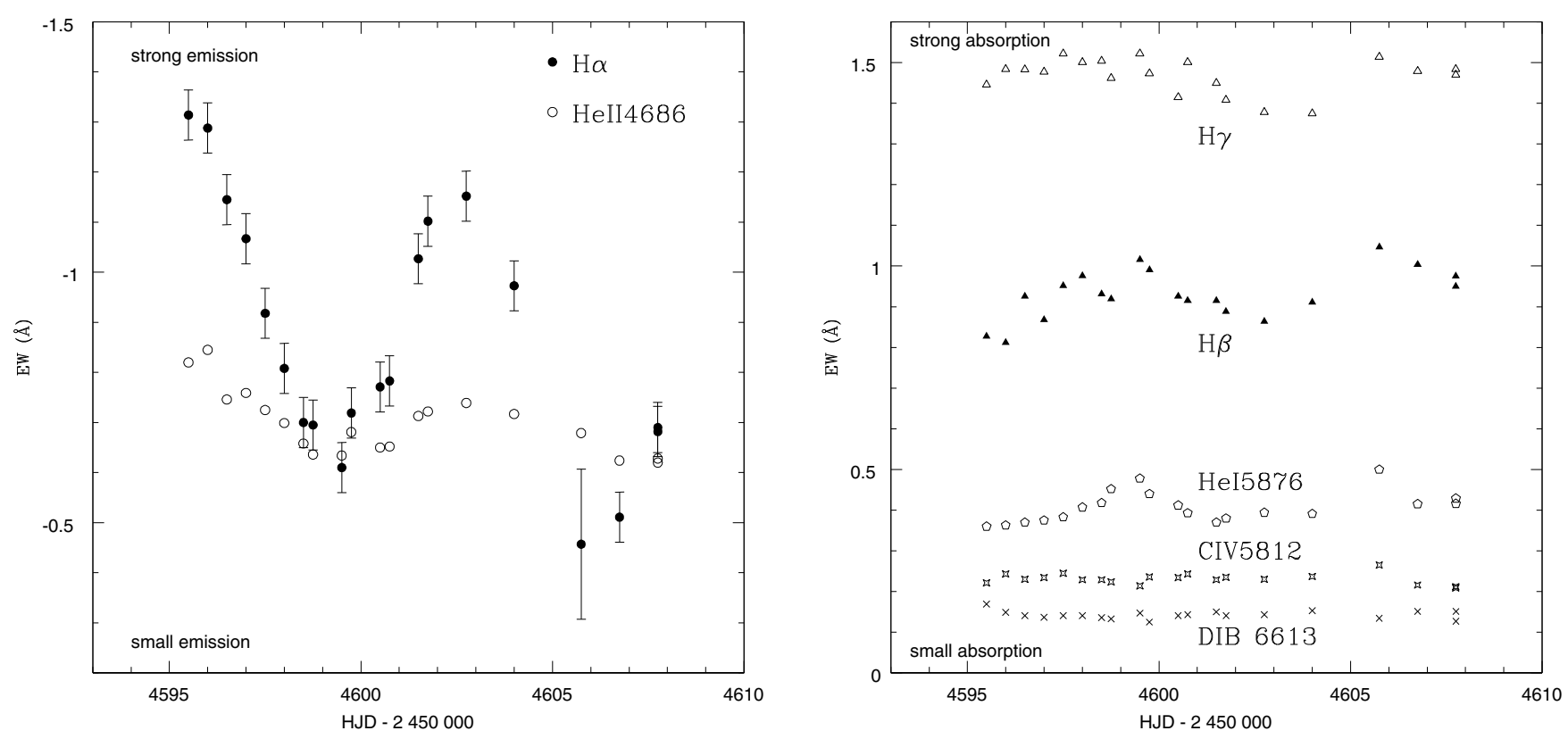

Fig. 3. Evolution with time of the $E W \mathrm{~s}$ (expressed in $\AA$ ) for some lines of HD 148937. Left: the strongest emission lines (Balmer line of H $\alpha$ and He II $\lambda$ 4686); the emission strength increases from bottom to top. Right: some absorption lines; the absorption increases from bottom to top. The $\mathrm{He} \mathrm{I} \lambda 5876$ and $\mathrm{H} \alpha$ data were corrected for the contamination by telluric lines.
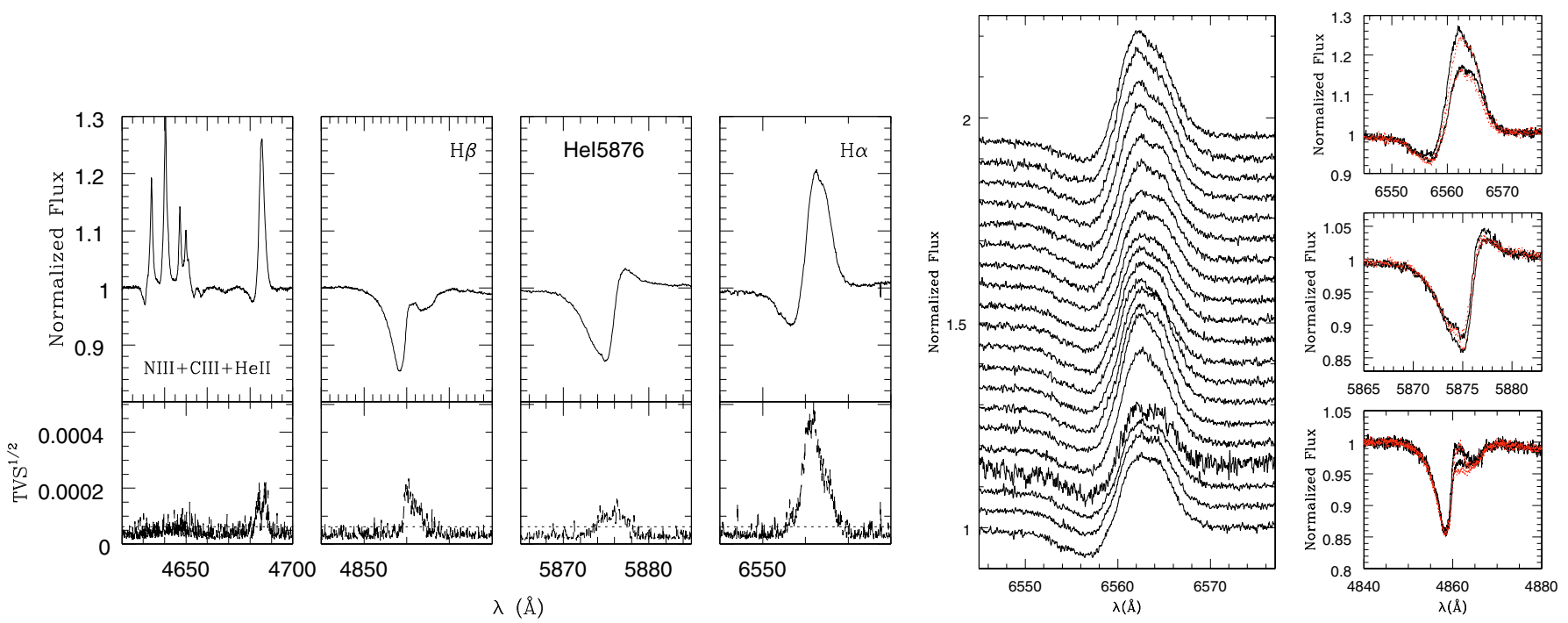

Fig. 4. Left: average spectra and TVS of He II $\lambda$ 4686, H $\beta$, He I $\lambda$ 5876, and $\mathrm{H} \alpha$ for HD 148937. The He I $\lambda 5876$ and $\mathrm{H} \alpha$ data were corrected for the contamination by telluric lines. The horizontal dotted line in the lower panels represents the $99 \%$ confidence level. Right: the 20 Coralie observations of the $\mathrm{H} \alpha$ line (from 2008 May 8 to May 21, note the high noise in the data of May 19, which is reflected in the $E W$ measurements) and the extreme states of $\mathrm{H} \alpha, \mathrm{He} \mathrm{I} \lambda 5876$, and $\mathrm{H} \beta$. The black solid lines correspond to the spectra of May 09 (max emission state) and May 12 ( $\mathrm{min}$ ), the dotted red lines to those of May $15(\max )$ and May $20(\mathrm{~min})$. The colored version of the figure is available on-line.

\section{3. $H D 191612$}

\subsubsection{X-ray data}

The previous observations of HD 191612 (Nazé et al. 2007) revealed changes in the $\mathrm{X}$-ray flux, but the origin of this variability was unclear. To get a better understanding of this phenomenon, we undertook a new XMM-Newton observation, whose scheduling was carefully chosen. While the previous observations were taken at orbital phases $\phi_{\text {orb }}=0.83-0.96$ and at phases $\phi_{\text {cyc }}=$ $0.09-0.44$ in the $538 \mathrm{~d}$ cycle, the new exposure corresponds to a phase $\phi_{\text {cyc }}=0.13$ in the line-profile/photometric cycle of $538 \mathrm{~d}$ and a phase $\phi_{\text {orb }}=0.55$ in the orbital cycle (Fig. 6, ephemeris taken from Howarth et al. 2007). The new data thus enable us to check whether the flux variations are linked to the circumstellar, possibly magnetic, structures surrounding the star or to colliding winds in a binary. In the former case, the new data should be nearly identical to the previous observation taken at a similar phase $\phi_{\text {cyc }}$. For the other case, a dramatic variation is expected though. In a colliding wind binary, variations of the flux, correlated with orbital phase, are expected to arise from changes 



Fig. 5. Left, from top to bottom: raw periodogram of $\mathrm{H} \alpha$ as observed during the 2008 May campaign, periodogram after prewhitening by the period of 7.16d, and associated spectral window in the case of HD 148937. The latter is directly related to the sampling of the time series. Right, from top to bottom: periodograms of $\mathrm{He}$ II $\lambda 4686, \mathrm{H} \beta$, and $\mathrm{He}$ I $\lambda$ 5876. The power spectra were calculated and averaged in the interval of significant variability (see Fig. 4 and top label of each panel). The $\mathrm{He} \mathrm{I} \lambda 5876$ and $\mathrm{H} \alpha$ data were corrected for the contamination by telluric lines.

in the separation (in eccentric systems) and/or in the absorbing column (when the winds are different). Indeed, such variations have been observed in several systems (for a review, see Güdel \& Nazé 2009). In HD 191612, the previous observations, taken before periastron, revealed a $20 \%$ change in reddening-corrected flux (see Table 2) for a phase difference $\Delta \phi_{\text {orb }}$ of only 0.12 , corresponding to a change in separation of $36 \%$. The new observation was taken two-thirds of the orbit later, near apastron: the change in separation compared to previous data has thus increased (up to $60 \%$ ), and so should the difference in flux, if it is truly linked to the colliding wind emission.

The new data were processed following the same procedure as for the previous data, but using a newer XMM-Newton reduction software. The count rates derived from a run of the task edetect_chain amount to $0.122 \pm 0.002 \mathrm{cts} \mathrm{s}^{-1}$ for EPIC MOS 1 , $0.119 \pm 0.002 \mathrm{cts} \mathrm{s}^{-1}$ for EPIC MOS2, and $0.392 \pm 0.005 \mathrm{cts} \mathrm{s}^{-1}$ for EPIC pn. The evolution of the count rates and hardness ratio is shown in Fig. 6. The new data agree very well with previous observations taken at the same phase of the $538 \mathrm{~d}$ cycle, both in strength (similar count rates) and in hardness (similar hardness ratios); they are in addition clearly different from the results of previous observations obtained at the latest phase (where the count rates are $\sim 35 \%$ smaller, corresponding to a $\sim 20 \sigma$ difference, and the hardness ratio $H R_{1}$ measured on pn data is lower by $7 \sigma)$.

The best spectral fit is provided in Table 2, together with the previous results for comparison (reproduced from Nazé et al. 2007). The fitted model has the form $\operatorname{wabs}\left(N_{\text {int }}^{\mathrm{H}}\right) * \operatorname{wabs}\left(N_{1}^{\mathrm{H}}\right) * \operatorname{mekal}\left(k T_{1}\right)+$ wabs $\left(N_{2}^{\mathrm{H}}\right) *$ mekal $\left.\left(k T_{2}\right)\right]$, with wabs $\left(N_{\text {int }}^{\mathrm{H}}\right)=3.4 \times 10^{21} \mathrm{~cm}^{-2}$ (Diplas \& Savage 1994) and using solar abundances for both absorptions and optically-thin plasma emissions - i.e. the same model as used in Nazé et al. (2007) for consistency reasons ${ }^{2}$. Quoted fluxes are in the $0.4-10.0 \mathrm{keV}$ energy range, and the unabsorbed fluxes $f_{\mathrm{X}}^{\text {unabs }}$ are corrected only for the interstellar absorbing column. For each parameter, the lower and upper limits of the $90 \%$ confidence interval (derived from the ERROR command under XSPEC) are noted as indices and exponents, respectively. The normalisation factors are defined as $\frac{10^{-14}}{4 \pi D^{2}} \int n_{\mathrm{e}} n_{\mathrm{H}} \mathrm{d} V$, where $D, n_{\mathrm{e}}$ and $n_{\mathrm{H}}$ are respectively the distance to the source, the electron and proton density of the emitting plasma. The phases $\phi_{\text {orb }}$ and $\phi_{\text {cyc }}$ correspond to the phases in the orbital cycle and the $538 \mathrm{~d}$ line profile/photometric cycle, respectively (ephemeris of Howarth et al. 2007). Again, the spectral fits to the 2008 data agree very well with the previous observations taken at the same phase of the $538 \mathrm{~d}$ cycle, yielding the same X-ray luminosity, $9 \times 10^{32} \mathrm{erg} \mathrm{s}^{-1}$ (for a distance of $2.29 \mathrm{kpc})$, and same $\log \left[L_{X} / L_{\mathrm{BOL}}\right],-6.1$.

The conclusions are obvious: despite the very different orbital phases, the new data are nearly identical to those taken three years before at a similar phase in the $538 \mathrm{~d}$ cycle. The modulation of the X-ray emission thus appears directly correlated with the optical line-profile variations, a situation very similar to that observed for $\theta^{1}$ Ori C (Gagné et al. 2005; Nazé et al. 2008b).

\subsubsection{MHD modelling}

To better understand the properties of HD 191612, a fullydynamic numerical magneto-hydrodynamic (MHD) model is needed that takes into account the impact on the wind of

\footnotetext{
2 As in Nazé et al. (2007), we noted that the two sets of parameters give a good fit, but we recall that a fit by a differential emission measure (DEM) model clearly favours the "cool" fit.
} 
Y. Nazé et al.: New findings on the prototypical Of?p stars

Table 2. Best-fitting models and X-ray fluxes at Earth for each XMM-Newton observation of HD 191612.

\begin{tabular}{|c|c|c|c|c|c|c|c|c|c|c|c|c|}
\hline Date & Rev. & $\phi_{\text {cyc }}$ & $\phi_{\text {orb }}$ & $\begin{array}{c}N_{1}^{\mathrm{H}} \\
10^{22} \mathrm{~cm}^{-2}\end{array}$ & $\begin{array}{l}k T_{1} \\
\mathrm{keV}\end{array}$ & $\begin{array}{c}\text { Norm }_{1} \\
10^{-3} \mathrm{~cm}^{-5}\end{array}$ & $\begin{array}{c}N_{2}^{\mathrm{H}} \\
10^{22} \mathrm{~cm}^{-2}\end{array}$ & $\begin{array}{l}k T_{2} \\
\mathrm{keV}\end{array}$ & $\begin{array}{c}\text { Norm }_{2} \\
10^{-3} \mathrm{~cm}^{-5}\end{array}$ & $\chi_{v}^{2}$ (d.o.f.) & $\begin{array}{l}f_{\mathrm{X}}^{\text {abs }} \\
\left(10^{-13}\right.\end{array}$ & $\begin{array}{c}f_{\mathrm{X}}^{\text {unabs }} \\
\left.\mathrm{g} \mathrm{cm}^{-2} \mathrm{~s}^{-1}\right)\end{array}$ \\
\hline \multicolumn{13}{|c|}{ "cool" model } \\
\hline 05/04/05 & 975 & 0.09 & 0.84 & $0.51_{0.42}^{0.61}$ & $0.23_{0.20}^{0.25}$ & $7.07_{4.04}^{15.9}$ & $1.07_{0.87}^{1.31}$ & $1.27_{1.19}^{1.36}$ & $1.08_{0.96}^{1.21}$ & $1.16(411)$ & 7.0 & 14.1 \\
\hline $17 / 04 / 05$ & 981 & 0.12 & 0.84 & $0.32_{0.25}^{0.46}$ & $0.27_{0.23}^{0.29}$ & $2.15_{1.40}^{3.81}$ & $0.91_{0.80}^{1.10}$ & $1.01_{0.96}^{1.07}$ & $1.22_{1.11}^{1.35}$ & $1.10(681)$ & 6.8 & 14.3 \\
\hline $02 / 06 / 05$ & 1004 & 0.20 & 0.87 & $0.43_{0.31}^{0.51}$ & $0.25_{0.23}^{0.28}$ & $3.78_{1.78}^{5.83}$ & $1.03_{0.81}^{1.25}$ & $1.22_{0.98}^{1.31}$ & $0.89_{0.74}^{1.111}$ & $1.15(460)$ & 6.0 & 12.5 \\
\hline 08/10/05 & 1068 & 0.44 & 0.96 & $0.61_{0.58}^{0.64}$ & $0.20_{0.19}^{0.21}$ & $13.6_{107}^{18.1}$ & $1.39_{1.14}^{1.68}$ & $1.60_{1.49}^{1.72}$ & $0.66_{0.59}^{0.76}$ & $1.31(555)$ & 5.3 & 11.0 \\
\hline 03/04/08 & 1523 & 0.13 & 0.55 & $0.51_{0.46}^{0.56}$ & $0.24_{0.23}^{0.25}$ & $6.67_{4.75}^{9.22}$ & $1.21_{1.05}^{1.44}$ & $1.34_{1.28}^{1.44}$ & $1.06_{0.97}^{1.17}$ & $1.31(591)$ & 7.2 & 14.4 \\
\hline \multicolumn{13}{|c|}{ "hot" model } \\
\hline 05/04/05 & 975 & 0.09 & 0.84 & 0.0 .05 & $0.64_{0.62}^{0.66}$ & $0.28_{0.27}^{0.33}$ & 0.0 .03 & $2.35_{2.21}^{2.54}$ & $0.51_{0.48}^{0.53}$ & $1.19(411)$ & 7.6 & 14.4 \\
\hline $17 / 04 / 05$ & 981 & 0.12 & 0.84 & 0.0 .01 & $0.60_{0.58}^{0.62}$ & $0.32_{0.31}^{0.31}$ & $0.81_{0.70}^{0.87}$ & $1.23_{1.06}^{1.321}$ & $0.95_{0.86}^{1.48}$ & $1.08(681)$ & 7.0 & 13.9 \\
\hline $02 / 06 / 05$ & 1004 & 0.20 & 0.87 & 0.0 .01 & $0.51_{0.47}^{0.58}$ & $0.28_{0.26}^{0.31}$ & $0.82_{0.74}^{0.91}$ & $\begin{array}{l}1.07_{1.01}^{1.20} \\
\end{array}$ & $0.92_{0.75}^{1.01}$ & $1.16(460)$ & 5.8 & 12.0 \\
\hline 08/10/05 & 1068 & 0.44 & 0.96 & 0.0 .01 & $0.61_{0.60}^{0.67}$ & $0.23_{0.22}^{0.20}$ & 0.0 .01 & $2.53_{2.38}^{2.68}$ & $0.32_{0.31}^{0.34}$ & $1.39(555)$ & 5.4 & 11.0 \\
\hline 03/04/08 & 1523 & 0.13 & 0.55 & 0.0 .01 & $0.61_{0.60}^{0.62}$ & $0.33_{0.32}^{0.35}$ & $0.57_{0.44}^{0.69}$ & $1.64_{1.53}^{1.76}$ & $0.73_{0.66}^{0.79}$ & $1.31(591)$ & 7.3 & 14.1 \\
\hline
\end{tabular}
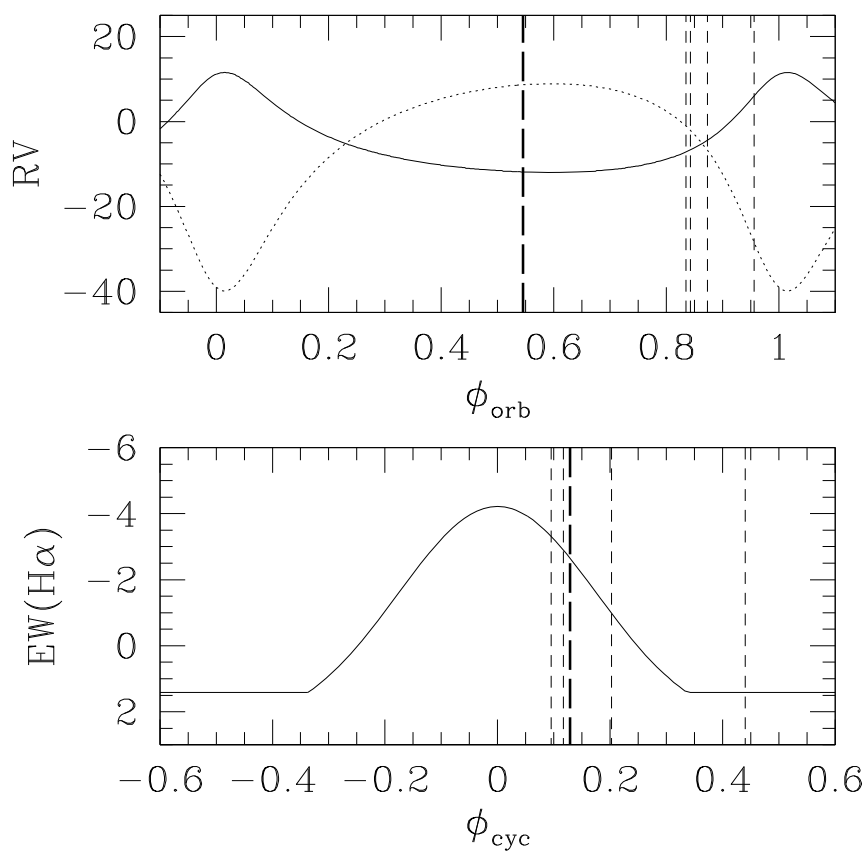


Fig. 6. Left: position of the new XMM-Newton observation in the cycles of HD 191612. The orbital cycle, calculated using orbital parameters of Howarth et al. (2007) and with the primary (resp. secondary) curve drawn in solid (resp. dotted) line, is shown on top while the bottom panel shows the line profile variation cycle of 538d from the analytical approximation of Howarth et al. (2007). The phases of the previous observations are shown by the thin short-dashed lines and the phase of the new observation by the thick long-dashed line. Right: evolution of the count rate in the 0.4-10.0 keV band (top) and of two hardness ratios (middle, bottom) with phase from the $538 \mathrm{~d}$ cycle. Open symbols refer to the old observations, filled red symbols to the new dataset; triangles and squares were used for the EPIC MOS data (note that the MOS count rates are multiplied by a factor of 3 in this figure) and circles for the EPIC pn data. The hardness ratio $H R_{1}$ and $H R_{2}$ are defined as $(M-S) /(M+S)$ and $(H-M) /(H+M)$, respectively, where $S$ is the count rate in the $0.4-1.0 \mathrm{keV}$ band, $M$ in $1-2 \mathrm{keV}$, and $H$ in $2-10 \mathrm{keV}$. The coloured version of the figure is available on-line.

the magnetic field detection. We used the publically available ZEUS-3D code (Stone \& Norman 1992) to evolve a consistent dynamical solution for a line-driven stellar wind from the star with a dipolar surface field. The stellar parameters of HD 191612, adopted mostly from Nazé et al. (2008b, and references therein), are listed in Table 3.

HD 191612 is a rather slow rotator with a surface rotation speed $<50 \mathrm{~km} \mathrm{~s}^{-1}$ (Howarth et al. 2007; Nazé et al. 2008b). This slow rotation probably has no significant dynamical effects on the stellar wind. Therefore, the wind was assumed to be azimuthally symmetric with the magnetic field axis aligned with the rotation axis ${ }^{3}$. This is essentially a " 2.5 -D" formulation allowing for non-zero azimuthal components of both the magnetic field $B_{\phi}$ and velocity $v_{\phi}$, while still assuming that all quantities are constant in the azimuthal coordinate angle $\phi$.

Much of the numerical procedures in this simulation follows ud-Doula et al. (2008). The models thus include rotational

3 This simplifies the computation and makes no assumption about the true geometry of the system, i.e. an oblique rotator model is not excluded; it can easily be simulated here by changing the viewing angle on the system. 
Table 3. Stellar parameters used for the MHD simulation of HD 191612, adopted mostly from Nazé et al. (2008b, and references therein).

\begin{tabular}{lr}
\hline \hline Parameter & Value \\
\hline$R$ & $1.015 \times 10^{12} \mathrm{~cm}=14.4 R_{\odot}$ \\
$M$ & $7.0 \times 10^{34} \mathrm{~g}=35 M_{\odot}$ \\
$L$ & $7.697 \times 10^{38} \mathrm{erg} \mathrm{s}^{-1}=2 \times 10^{5} L_{\odot}$ \\
$\alpha$ & 0.55 \\
$\bar{Q}$ & 2000 \\
$\delta$ & 0.0 \\
$\dot{M}$ & $1 \times 10^{-6} M_{\odot} \mathrm{yr}^{-1}$ \\
$v_{\text {rot }}$ & $50 \mathrm{~km} \mathrm{~s}^{-1}$ \\
$B_{\text {polar }}$ & $1500 \mathrm{G}$ \\
$\eta_{*}$ & $31 \sim \sqrt{1000}$ \\
\hline
\end{tabular}

effects, but for the first time we also use the full energy equation, with an adiabatic index $\gamma=5 / 3$ and a radiative cooling of MacDonald \& Bailey (1981, who assumed solar abundance $)^{4}$. At the initial time $t=0$, the wind is represented by a relaxed non-magnetic and non-rotating model, but we then simultaneously introduce a dipole magnetic field and a surface rotation at the lower boundary, both defined relative to a common polar axis. The wind and magnetic field are then let free to compete with each other. To ensure that there are no numerical artefacts arising from the initial conditions, we run our model for about $1.5 \mathrm{Msec}$, which is at least 50 fluid crossing times.

Immediately after $t=0$, the magnetic field channels wind material towards the tops of closed loops near the equator. There the collision with the opposite stream leads to strong shocks, like in "magnetically confined wind shocks" (MCWS) presented by Babel \& Montmerle (1997). Denser material then cools radiatively very quickly, leading to a dense disc-like structure (Fig. 7). Some of the less dense post-shock material within the closed magetic loops remain very hot (ca. 80MK) for a long period of time. In this model, the Alfvén radius or magnetosphere extends about 3-4 stellar radii above the stellar surface.

Initially mass builds up in the equatorial region below the Keplerian radius estimated to be about $4.5 R_{*}$, but then there appear repeated episodes of infall of inner disc material back onto the stellar surface. Over a longer timescale, there appears another, somewhat different kind of disruption, one that starts higher up, closer to the Alfvén radius. This is characterized by outward ejections of the upper disc mass, leading to "centrifugally driven mass ejections". These ejections can lead to X-ray flares (see ud-Doula et al. 2006), but these short-term variations cannot be detected in the observations of HD 191612, since they lack the required sensitivity to analyse the lightcurve on very short timescales.

Figure 8 shows the differential emission measure (DEM, or the relative amount of material in a given temperature bin) calculated in the MHD simulation. The DEM peaks at very high temperatures (about $10 \mathrm{MK}$ and larger), suggesting that the very hot gas dominates the X-ray emission of the system. Of course, the material in the unshocked regions remains at the effective temperature of the star due to the intense UV radiation from the parent star that keeps the wind nearly isothermal.

\footnotetext{
${ }^{4}$ Note that models used by Gagné et al. (2005) made use of the full energy equation, but did not include rotation.
}

\subsubsection{Comparison of models and observations}

Applying a magnetically-confined wind model to HD 191612 yields four clues. First, the X-ray emission should clearly be dominated by very hot plasma, as was the case for $\theta^{1}$ Ori C (see Fig. 6 of Gagné et al. 2005). Second, on average, the total EM calculated for HD191612 in the 1-100 MK range amounts to $5.3 \times 10^{56} \mathrm{~cm}^{-3}$. This agrees well with the MHD simulation of $\theta^{1}$ Ori $\mathrm{C}$, considering the difference in the stellar parameters and modelling. Indeed, the simulated EM for this star in the same temperature range reached $8.2 \times 10^{55} \mathrm{~cm}^{-3}$ (Gagné et al. 2005), i.e. a factor 6.5 lower than for HD 191612, which is close to the factor of 4 expected from the EMs scaling with the third power of the radius. Considering an average cooling factor $\Lambda(T)$ of $\sim 2 \times 10^{-23} \mathrm{erg} \mathrm{cm}^{3} \mathrm{~s}^{-1}$ (Raymond et al. 1976), this yields a totally unabsorbed X-ray luminosity of $\sim 8 \times 10^{33} \mathrm{erg} \mathrm{s}^{-1}$ in the $0.5-10 . \mathrm{keV}$ range. Third, the hot gas generally moves with velocities of about $100-200 \mathrm{~km} \mathrm{~s}^{-1}$ in the MHD models and only very few hot plasma reach $400 \mathrm{~km} \mathrm{~s}^{-1}$, whatever the viewing angle on the equatorially-confined region. This is unsurprising because the hot gas always remains close to the star: the associated $\mathrm{X}$-ray lines are thus expected to be narrow, again a similar result to that found for $\theta^{1}$ Ori C. Finally, the X-ray emission should be modulated throughout the cycle of 538d, because the viewing angle on the disc-like structure changes.

The observations (both old and new) do not fully agree with this picture. On the one hand, the emission is modulated with the 538d cycle, as expected for MCWS. The observed X-ray emission is indeed bright $\left(L_{X}=7-9 \times 10^{32} \mathrm{erg} \mathrm{s}^{-1}\right.$ when corrected for the interstellar absorption), it is on the luminous edge compared to "normal" O-type stars (for O stars observed with XMMNewton, $\log \left[L_{X} / L_{\mathrm{BOL}}\right]=-6.45$ with a dispersion of $0.51 \mathrm{dex}$, see Nazé 2009). Considering the uncertainty in the spectral models (see Table 2), the observations yield a totally unabsorbed X-ray luminosity of $\sim 8-80 \times 10^{32} \mathrm{erg} \mathrm{s}^{-1}$, for the "hot" and "cool" model respectively. Total emission measures, derived from the normalisation factors of Table 2 or as in Gagné et al. (2005) for a spectral fitting considering a single absorbing column for both thermal components, amount to $3-7 \times 10^{56} \mathrm{~cm}^{-3}$ for an equivalent "cool" model and ten times lower for an equivalent "hot" model. Theoretical predictions thus agree rather well with the "cool" model, which is also the one favored by the DEM modelling (Nazé et al. 2007).

On the other hand, however, the X-ray emission of HD 191612 displays broad lines $\left(F W H M_{\text {obs }} \sim 2000 \mathrm{~km} \mathrm{~s}^{-1}\right.$, Nazé et al. 2007) and is far from being as hard as predicted by the model. Indeed, a fit of the EPIC data by a DEM model yields a strong emission peak at about $2 \mathrm{MK}$, not beyond $10 \mathrm{MK}$, as shown for example in Fig. 3 of Nazé et al. (2007). In addition, the average temperature ${ }^{5}$ derived in the $2 \mathrm{XMM}$ survey for HD 191612 amounts to $\sim 0.3 \mathrm{keV}$ and is thus quite normal $(85 \%$ of the stars in the 2XMM have $\langle k T\rangle$ below $1 \mathrm{keV}$, Nazé 2009) it does not resemble the extreme case of $\theta^{1}$ Ori $\mathrm{C}$ for which $\langle k T\rangle$ amounts to $2.5 \mathrm{keV}$ in the same $2 \mathrm{XMM}$ survey and for which the MCWS model works well. The relatively soft character of that emission despite the large magnetic confinement parameter might be reminiscent of the characteristics observed in some B-type stars (Güdel \& Nazé 2009): further investigation of the MCWS model and magnetic field geometry is thus required to understand the high-energy properties of the Of?p stars.

\footnotetext{
$5 \sum k T_{i} \times \operatorname{norm}_{i} / \sum$ norm $_{i}$ for models using a single absorption in front of the thermal emission components.
} 

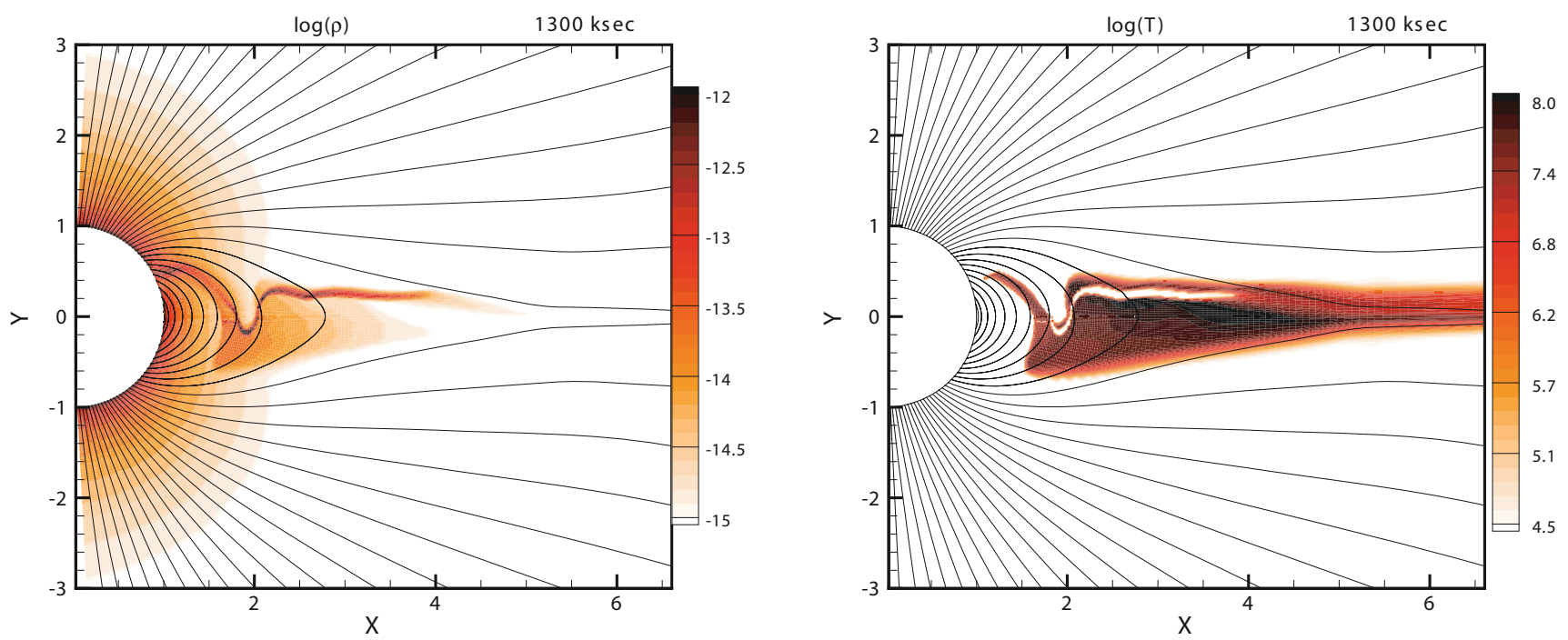

Fig. 7. Snapshots of density (left) and temperature (right), in cgs units on a logarithmic colour-scale, shown with field lines (solid lines) at arbitrarily chosen time $t=1300 \mathrm{ks}$ for the 2D MHD simulation of HD 191612. The $x$ and $y$ axes are labelled in units of the stellar radius. Note the unsteady nature of the dense equatorial region, which undergoes episodic emptyings and refills. Within the magnetosphere (i.e. the closed loop region), the opacity at $10 \AA$ or $1.2 \mathrm{keV}$ may reach high values $\left(\tau=10\right.$ for $\kappa=30 \mathrm{~cm}^{2} \mathrm{~g}^{-1}$, see Cohen 2009 , a density of $10^{-12} \mathrm{~g} \mathrm{~cm}^{-3}$ and a disc height of $0.03 R_{*}$ ), but it is totally negligible in the dense equatorial region outside the magnetosphere. The observations show only a small absorption in addition to the interstellar extinction, and it is not significantly varying with phase (i.e. there is no clear evidence of excess absorption in the magnetic equatorial plane, or the effect is much smaller than would be expected from a dense equatorial cooling disc, as found in Gagné et al. 2005).
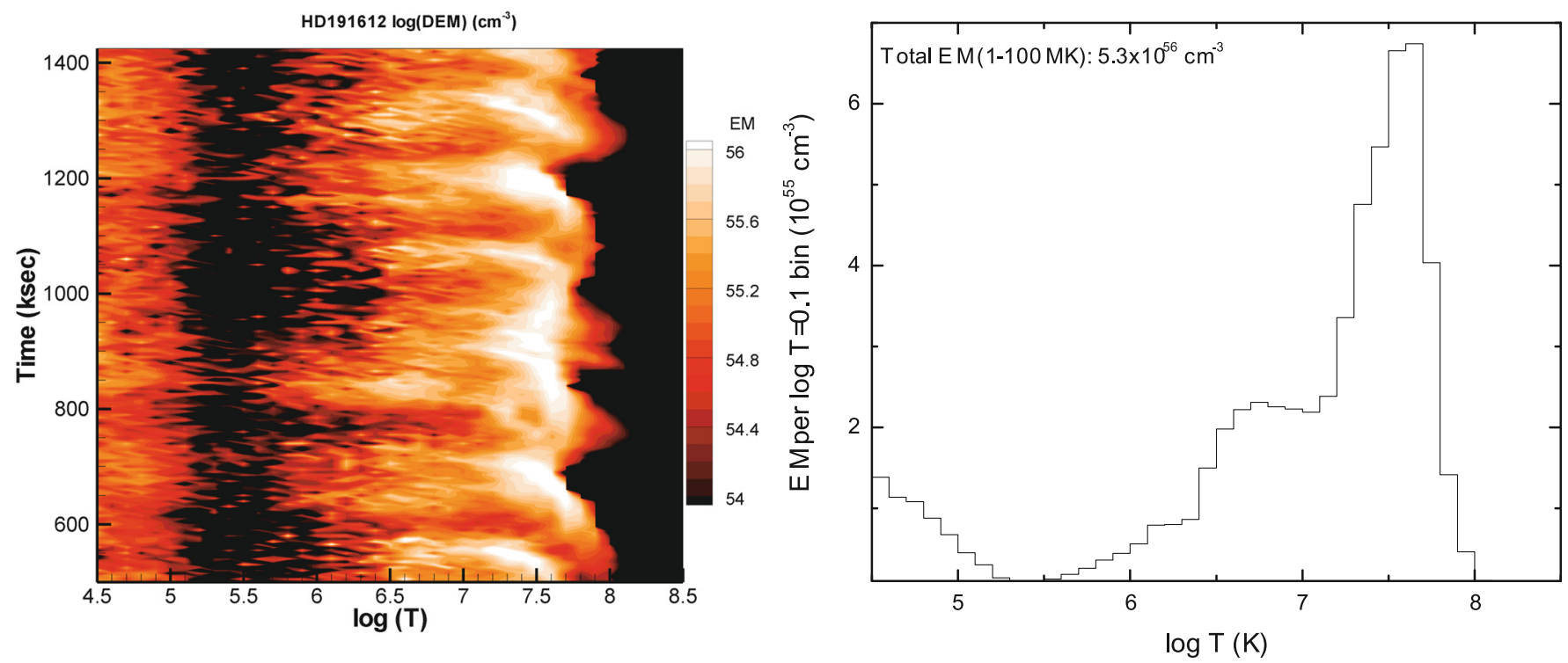

Fig. 8. Volume emission-measure distribution (DEM) per $\log T=0.1$ bin for our MHD simulations of HD 191612. The MHD simulation is used to compute the emission measure per unit volume per logarithmic temperature bin, then integrated over three-dimensional space assuming azimuthal symmetry about the magnetic dipole axis. Left: DEM as a function of time and temperature; the periodic variations due to build-ups and infalls are clearly seen. The coloured version of this figure is available on-line. Right: time-averaged DEM as a function of temperature.

\section{Conclusions}

Our continued monitoring in the X-ray and optical domains of the prototypical Of?p stars has now clarified some of their observational properties.

For HD 108, the Balmer, C III 24650 , He I, and Si III lines display minimum emissions since 2005: the star has thus finally reached its quiescent state for the first time in 50-60 yr. Longterm monitoring of this quiescence will further constrain the geometry of the line emission region.

For HD 148937, the $7 d$ variations in the Balmer lines are confirmed by a short-term monitoring. During this cycle, the amplitude of the emission peak in $\mathrm{H} \alpha$, the most variable line, changes by only $7 \%$. Thanks to the high spectral resolution of 
the new data, similar periodic variations, though of even smaller amplitude, are detected in the He I $\lambda 5876$ and He II $\lambda 4686$ lines. These detections underline the similarities of the star with the other two prototypical Of?p objects.

For HD 191612, a new XMM-Newton observation clearly shows that its X-ray emission is modulated by the $538 \mathrm{~d}$ period and not the orbital period - it is thus not of colliding-wind origin: the phenomenon responsible for the optical changes appears also at work in the high-energy domain. Contrary to observations, our MHD simulations predict a hard spectrum, dominated by very hot plasma ( $>10 \mathrm{MK})$ and narrow lines. Further refinements to the modelling will be needed in order to fully explain this discrepancy.

Acknowledgements. Y.N., M.D.B. and G.R. acknowledge support from the Fonds National de la Recherche Scientifique (Belgium), the Communauté Française de Belgique, the PRODEX XMM and Integral contracts, and the "Action de Recherche Concertée" (CFWB-Académie Wallonie Europe). The authors thank L. Mahy and P. Eenens for providing the 2009 échelle spectrum. ADS and CDS were used for preparing this document.

\section{References}

Babel, J., \& Montmerle, T. 1997, A\&A, 323, 121

Baranne, A., Queloz, D., Mayor, M., et al. 1996, A\&AS, 119, 373

Cohen, D. H. 2009, AIP Conf. Ser., 1161, 132

Diplas, A., \& Savage, B. D. 1994, ApJS, 93, 211

Donati, J.-F., Howarth, I. D., Bouret, J.-C., et al. 2006, MNRAS, 365, L6

Fullerton, A. W., Gies, D. R., \& Bolton, C. T. 1996, ApJS, 103, 475

Gagné, M., Oksala, M. E., Cohen, D. H., et al. 2005, ApJ, 628, 986
Gosset, E., Royer, P., Rauw, G., Manfroid, J., \& Vreux, J.-M. 2001, MNRAS, 327,435

Güdel, M., \& Nazé, Y. 2009, A\&ARv, 17, 309

Heck, A., Manfroid, J., \& Mersch, G. 1985, A\&AS, 59, 63

Hinkle, K., Wallace, L., Valenti, J., \& Harmer, D. 2000, Visible and Near Infrared Atlas of the Arcturus Spectrum 3727-9300 A, ed. K. Hinkle, L. Wallace, J. Valenti, \& D. Harmer (San Francisco: ASP)

Howarth, I. D., Walborn, N. R., Lennon, D. J., et al. 2007, MNRAS, 381, 433

Hubrig, S., Schöller, M., Schnerr, R. S., et al. 2008, A\&A, 490, 793

MacDonald, J., \& Bailey, M. E. 1981, MNRAS, 197, 995

Martins, F., Donati, J.-F., Marcolino, W. L. F., et al. 2010, MNRAS, 407, 1423

Nazé, Y. 2009, A\&A, 506, 1055

Nazé, Y., Vreux, J.-M., \& Rauw, G. 2001, A\&A, 372, 195

Nazé, Y., Rauw, G., Vreux, J.-M., \& De Becker, M. 2004, A\&A, 417, 667

Nazé, Y., Barbieri, C., Segafredo, A., Rauw, G., \& De Becker, M. 2006, IBVS, 5693

Nazé, Y., Rauw, G., Pollock, A. M. T., Walborn, N. R., \& Howarth, I. D. 2007, MNRAS, 375, 145

Nazé, Y., Walborn, N. R., Rauw, G., et al. 2008a, AJ, 135, 1946

Nazé, Y., Walborn, N. R., \& Martins, F. 2008b, Rev. Mex. Astron. Astrofis., 44, 331

Rauw, G., Morrison, N. D., Vreux, J.-M., Gosset, E., \& Mulliss, C. L. 2001, A\&A, 366, 585

Raymond, J. C., Cox, D. P., \& Smith, B. W. 1976, ApJ, 204, 290

Stone, J. M., \& Norman, M. L. 1992, ApJS, 80, 753

ud-Doula, A., \& Owocki, S. P. 2002, ApJ, 576, 413

ud-Doula, A., Townsend, R. H. D., \& Owocki, S. P. 2006, ApJ, 640, L191

ud-Doula, A., Owocki, S. P., \& Townsend, R. H. D. 2008, MNRAS, 385, 97

ud-Doula, A., Owocki, S. P., \& Townsend, R. H. D. 2009, MNRAS, 392, 1022

Walborn, N. R. 1972, AJ, 77, 312

Walborn, N. R., Howarth, I. D., Herrero, A., \& Lennon, D. J. 2003, ApJ, 588, 1025

Walborn, N. R., Howarth, I. D., Rauw, G., et al. 2004, ApJ, 617, L61

Walborn, N. R., Sota, A., Maiz-Apellaniz, J., et al. 2010, ApJ, 715, L74 